\title{
Secondary organic material formed by methylglyoxal in aqueous aerosol mimics. Part II: Product identification using Aerosol-CIMS
}

\author{
N. Sareen, E. L. Shapiro, A. N. Schwier, and V. F. McNeill*
}

\{Department of Chemical Engineering, Columbia University, New York, New York, USA\}

Correspondence to: V. F. McNeill (vfm2103@columbia.edu)

\section{SUPPLEMENTARY MATERIAL}

Table S1. B3LYP/ERMLER2 calculated total Gibbs free energies. Labels refer to Table 2 in the text. "cluster" denotes the cluster of the species with I.

\begin{tabular}{|l|r|}
\hline Species & $\mathbf{G}_{\text {tot }}$ (Hartrees) \\
\hline $\mathrm{I}^{-}$ & -111.5214881 \\
\hline $\mathrm{I}^{\cdot} \mathrm{H}_{2} \mathrm{O}$ & -187.9354270 \\
\hline $\mathrm{H}_{2} \mathrm{O}$ & -76.3838860 \\
\hline $\mathrm{HI}$ & -112.0452540 \\
\hline (a) & -267.0245790 \\
\hline (a) cluster & -378.5773160 \\
\hline (b) & -343.4048790 \\
\hline (b) cluster & -454.9726860 \\
\hline (c) & -457.6584990 \\
\hline (c) cluster & -569.2161910 \\
\hline (d) & -534.0437570 \\
\hline (d) cluster & -645.6083500 \\
\hline (e) & -534.0793570 \\
\hline (e) cluster & -645.6313070 \\
\hline (f) & -534.0829650 \\
\hline (f) cluster & -645.6532510 \\
\hline (g) & -494.3230970 \\
\hline (g) cluster & -605.8775960 \\
\hline (h) & -610.4375880 \\
\hline (h) cluster & -722.0139790 \\
\hline (i) & -610.4236450 \\
\hline (i) cluster & -722.0002230 \\
\hline (j) & -1158.7736880 \\
\hline (j) ionized & -1158.2948550 \\
\hline
\end{tabular}


Table S2. Cartesian coordinates for B3LYP/ERMLER2 optimized geometries. Labels refer to Table 2 in the text. "cluster" denotes the cluster of the species with I'.

$\begin{array}{rrrr}\mathrm{H}_{2} \mathrm{O} & & & \\ \mathrm{O} 1 & 0.1066000000 & -1.6372000000 & 0.0000000000 \\ \mathrm{H} 2 & 1.0481000000 & -1.3803000000 & 0.0000000000 \\ \mathrm{H} 3 & -0.4328000000 & -0.8238000000 & 0.0000000000\end{array}$

\section{$\Gamma \cdot \mathrm{H}_{2} \mathrm{O}$}

$\begin{array}{llll}\text { I1 } & -0.3414000000 & 1.9392000000 & 0.0000000000 \\ \text { O2 } & 0.1066000000 & -1.6372000000 & 0.0000000000 \\ \text { H3 } & 1.0494000000 & -1.3800000000 & 0.0000000000 \\ \text { H4 } & -0.3685000000 & -0.7660000000 & 0.0000000000\end{array}$

HI

I1 $\quad-0.7431000000 \quad 0.1665000000 \quad 0.0000000000$

$\mathrm{H} 2 \quad 0.8869000000 \quad-0.1988000000 \quad 0.0000000000$

(a)

$\begin{array}{lrrr}\text { C1 } & -0.7453000000 & 0.0567000000 & 0.0011000000 \\ \text { C2 } & 0.7906000000 & -0.0118000000 & 0.0006000000 \\ \text { O3 } & -1.2534000000 & 1.0888000000 & -0.3731000000 \\ \text { C4 } & -1.4938000000 & -1.1584000000 & 0.4668000000 \\ \text { H5 } & 1.2635000000 & 0.9249000000 & -0.3479000000 \\ \text { O6 } & 1.4136000000 & -0.9820000000 & 0.3416000000 \\ \text { H7 } & -1.1964000000 & -2.0270000000 & -0.1225000000 \\ \text { H8 } & -2.5635000000 & -0.9899000000 & 0.3860000000 \\ \text { H9 } & -1.2226000000 & -1.3828000000 & 1.5001000000\end{array}$

\section{(a) cluster}

$\begin{array}{llll}\text { C1 } & -0.7453000000 & 0.0567000000 & 0.0011000000 \\ \text { C2 } & 0.7649000000 & -0.0107000000 & 0.0006000000 \\ \text { O3 } & -1.3057000000 & 1.0813000000 & -0.4427000000 \\ \text { C4 } & -1.4930000000 & -1.2001000000 & 0.3737000000 \\ \text { H5 } & 1.2583000000 & 0.9698000000 & -0.0198000000 \\ \text { O6 } & 1.3790000000 & -1.0831000000 & -0.1895000000 \\ \text { I7 } & 0.4564000000 & 0.7586000000 & 3.1088000000 \\ \text { H8 } & -1.0391000000 & -2.0639000000 & -0.1244000000 \\ \text { H9 } & -2.5459000000 & -1.1019000000 & 0.0984000000 \\ \text { H10 } & -1.4030000000 & -1.3546000000 & 1.4539000000\end{array}$

(b)

$\begin{array}{lrrr}\text { C1 } & -0.7165000000 & 0.2340000000 & 0.0711000000 \\ \text { C2 } & 0.5642000000 & 1.0706000000 & 0.1203000000 \\ \text { O3 } & -1.3225000000 & 0.1211000000 & -1.0030000000 \\ \text { C4 } & -1.1894000000 & -0.3990000000 & 1.3547000000 \\ \text { H5 } & -1.4353000000 & 0.3672000000 & 2.1024000000 \\ \text { O6 } & 0.3625000000 & 2.3199000000 & 0.8078000000\end{array}$




$\begin{array}{lccc}\text { O7 } & 1.5517000000 & 0.2606000000 & 0.7748000000 \\ \text { H8 } & 0.8461000000 & 1.3545000000 & -0.8952000000 \\ \text { H9 } & 0.1438000000 & 2.1509000000 & 1.7493000000 \\ \text { H10 } & 2.4340000000 & 0.6803000000 & 0.7162000000 \\ \text { H11 } & -0.3957000000 & -1.0245000000 & 1.7758000000 \\ \text { H12 } & -2.0821000000 & -0.9953000000 & 1.1596000000\end{array}$

\section{(b) cluster}

$\begin{array}{lccc}\text { C1 } & -0.7165000000 & 0.2340000000 & 0.0711000000 \\ \text { C2 } & 0.5715000000 & 1.0754000000 & 0.1206000000 \\ \text { O3 } & -1.2775000000 & 0.0512000000 & -1.0256000000 \\ \text { C4 } & -1.2492000000 & -0.3446000000 & 1.3550000000 \\ \text { H5 } & -1.3116000000 & 0.4209000000 & 2.1411000000 \\ \text { O6 } & 0.2437000000 & 2.4678000000 & 0.2425000000 \\ \text { O7 } & 1.4814000000 & 0.5959000000 & 1.1222000000 \\ \text { H8 } & 1.0701000000 & 0.9655000000 & -0.8437000000 \\ \text { H9 } & -0.0075000000 & 2.6828000000 & 1.1837000000 \\ \text { H10 } & 1.2506000000 & 0.9883000000 & 2.0048000000 \\ \text { H11 } & -0.5543000000 & -1.1090000000 & 1.7242000000 \\ \text { H12 } & -2.2297000000 & -0.7921000000 & 1.1765000000 \\ \text { I13 } & -0.0457000000 & 2.6698000000 & 3.8294000000\end{array}$

(c)

$\begin{array}{llcc}\text { C1 } & -0.6294000000 & 0.4713000000 & -0.0359000000 \\ \text { C2 } & 0.5864000000 & -0.3587000000 & 0.1030000000 \\ \text { O3 } & -0.6806000000 & 1.5497000000 & -0.5927000000 \\ \text { C4 } & -1.9291000000 & -0.0783000000 & 0.5733000000 \\ \text { H5 } & -2.7905000000 & 0.5971000000 & 0.4241000000 \\ \text { H6 } & 1.8308000000 & 0.9766000000 & -0.9231000000 \\ \text { C7 } & 1.7593000000 & 0.0307000000 & -0.3994000000 \\ \text { H8 } & 0.5006000000 & -1.3001000000 & 0.6279000000 \\ \text { C9 } & 2.9938000000 & -0.8096000000 & -0.2597000000 \\ \text { O10 } & -2.0056000000 & -1.1297000000 & 1.1509000000 \\ \text { C11 } & 4.2562000000 & -0.2446000000 & -0.8657000000 \\ \text { O12 } & 2.9625000000 & -1.8799000000 & 0.3097000000 \\ \text { H13 } & 5.0884000000 & -0.9209000000 & -0.6963000000 \\ \text { H14 } & 4.4768000000 & 0.7334000000 & -0.4313000000 \\ \text { H15 } & 4.1178000000 & -0.0884000000 & -1.9383000000\end{array}$

\section{(c) cluster}

$\begin{array}{lrrr}\text { C1 } & -0.6294000000 & 0.4713000000 & -0.0359000000 \\ \text { C2 } & 0.5718000000 & -0.3487000000 & 0.1013000000 \\ \text { O3 } & -0.6215000000 & 1.6877000000 & 0.2712000000 \\ \text { C4 } & -1.9028000000 & -0.1461000000 & -0.6015000000 \\ \text { H5 } & -1.7947000000 & -1.0049000000 & -1.2877000000 \\ \text { H6 } & -0.2286000000 & -2.2877000000 & -0.3605000000 \\ \text { C7 } & 0.6294000000 & -1.6916000000 & -0.0590000000 \\ \text { H8 } & 1.4751000000 & 0.1883000000 & 0.3820000000\end{array}$




$\begin{array}{lccc}\text { C9 } & 1.9106000000 & -2.4266000000 & 0.1316000000 \\ \text { O10 } & -3.0150000000 & 0.3277000000 & -0.3291000000 \\ \text { C11 } & 1.8733000000 & -3.9153000000 & -0.0848000000 \\ \text { O12 } & 2.9618000000 & -1.8347000000 & 0.4653000000 \\ \text { H13 } & 2.8817000000 & -4.3288000000 & -0.0182000000 \\ \text { H14 } & 1.4103000000 & -4.1474000000 & -1.0536000000 \\ \text { H15 } & 1.2349000000 & -4.3886000000 & 0.6748000000 \\ \text { I16 } & -1.1192000000 & -3.6353000000 & -2.7734000000\end{array}$

(d)

$\begin{array}{llcc}\text { C1 } & -0.6294000000 & 0.4713000000 & -0.0359000000 \\ \text { C2 } & 0.6051000000 & -0.3715000000 & 0.1051000000 \\ \text { O3 } & -0.6538000000 & 1.5776000000 & -0.6125000000 \\ \text { C4 } & -1.9330000000 & -0.0270000000 & 0.5633000000 \\ \text { H5 } & -2.7806000000 & 0.6632000000 & 0.4304000000 \\ \text { O6 } & 2.1810000000 & 1.4494000000 & -0.4612000000 \\ \text { C7 } & 1.7497000000 & 0.1088000000 & -0.7974000000 \\ \text { H8 } & 1.4073000000 & 0.0802000000 & -1.8469000000 \\ \text { C9 } & 2.9601000000 & -0.8236000000 & -0.7096000000 \\ \text { O10 } & -2.0303000000 & -1.1097000000 & 1.1464000000 \\ \text { C11 } & 4.3276000000 & -0.2096000000 & -0.8171000000 \\ \text { O12 } & 2.7744000000 & -2.0455000000 & -0.5810000000 \\ \text { H13 } & 5.0846000000 & -0.9950000000 & -0.7911000000 \\ \text { H14 } & 4.4809000000 & 0.5006000000 & 0.0025000000 \\ \text { H15 } & 4.4178000000 & 0.3732000000 & -1.7413000000 \\ \text { H16 } & 0.3785000000 & -1.4258000000 & -0.0891000000 \\ \text { H17 } & 0.9282000000 & -0.3342000000 & 1.1569000000 \\ \text { H18 } & 1.4401000000 & 2.0634000000 & -0.6512000000\end{array}$

\section{(d) cluster}

$\begin{array}{llcc}\text { C1 } & -0.6294000000 & 0.4713000000 & -0.0359000000 \\ \text { C2 } & 0.6081000000 & -0.3735000000 & 0.1055000000 \\ \text { O3 } & -1.1364000000 & 0.7540000000 & -1.1357000000 \\ \text { C4 } & -1.2639000000 & 0.9912000000 & 1.2552000000 \\ \text { H5 } & -0.9494000000 & 0.4970000000 & 2.1864000000 \\ \text { O6 } & 1.6488000000 & 1.2099000000 & 1.7188000000 \\ \text { C7 } & 1.8351000000 & 0.5072000000 & 0.4751000000 \\ \text { H8 } & 1.9371000000 & 1.2952000000 & -0.2833000000 \\ \text { C9 } & 3.1343000000 & -0.3134000000 & 0.4134000000 \\ \text { O10 } & -2.1003000000 & 1.9028000000 & 1.2407000000 \\ \text { C11 } & 4.0713000000 & -0.2381000000 & 1.5858000000 \\ \text { O12 } & 3.3774000000 & -0.9954000000 & -0.6001000000 \\ \text { H13 } & 5.0129000000 & -0.7316000000 & 1.3362000000 \\ \text { H14 } & 3.6047000000 & -0.7386000000 & 2.4491000000 \\ \text { H15 } & 4.2374000000 & 0.8016000000 & 1.8863000000 \\ \text { H16 } & 0.8130000000 & -0.8834000000 & -0.8386000000 \\ \text { H17 } & 0.4765000000 & -1.1124000000 & 0.9099000000 \\ \text { H18 } & 1.4923000000 & 0.5614000000 & 2.4669000000\end{array}$


(e)

$\begin{array}{llcc}\text { C1 } & -0.4148000000 & 0.6649000000 & -0.0252000000 \\ \text { C2 } & 0.4457000000 & -0.5213000000 & 0.0478000000 \\ \text { O3 } & -0.0136000000 & 1.7597000000 & 0.4709000000 \\ \text { C4 } & -1.7522000000 & 0.5460000000 & -0.6902000000 \\ \text { O5 } & 2.2153000000 & 0.6566000000 & 1.2054000000 \\ \text { C6 } & 1.6910000000 & -0.4800000000 & 0.6425000000 \\ \text { O7 } & -0.0788000000 & -1.6580000000 & -0.5147000000 \\ \text { C8 } & 2.5509000000 & -1.6666000000 & 0.7141000000 \\ \text { H9 } & 0.5818000000 & -2.4039000000 & -0.4117000000 \\ \text { H10 } & -2.2639000000 & 1.5083000000 & -0.6525000000 \\ \text { H11 } & -2.3567000000 & -0.2246000000 & -0.1984000000 \\ \text { H12 } & -1.6331000000 & 0.2220000000 & -1.7306000000 \\ \text { C13 } & 3.8911000000 & -1.5503000000 & 1.3742000000 \\ \text { O14 } & 2.1459000000 & -2.7607000000 & 0.2196000000 \\ \text { H15 } & 4.4000000000 & -2.5141000000 & 1.3361000000 \\ \text { H16 } & 3.7772000000 & -1.2243000000 & 2.4145000000 \\ \text { H17 } & 4.4963000000 & -0.7826000000 & 0.8787000000 \\ \text { H18 } & 1.5531000000 & 1.4010000000 & 1.1049000000\end{array}$

\section{(e) cluster}

$\begin{array}{lrrr}\text { C1 } & -0.4148000000 & 0.6649000000 & -0.0252000000 \\ \text { C2 } & 0.4438000000 & -0.5187000000 & 0.0476000000 \\ \text { O3 } & -0.0741000000 & 1.7640000000 & 0.5345000000 \\ \text { C4 } & -1.7208000000 & 0.5493000000 & -0.7582000000 \\ \text { O5 } & 2.0791000000 & 0.6568000000 & 1.3326000000 \\ \text { C6 } & 1.6341000000 & -0.4880000000 & 0.7391000000 \\ \text { O7 } & 0.0603000000 & -1.6381000000 & -0.6767000000 \\ \text { C8 } & 2.5651000000 & -1.6485000000 & 0.9120000000 \\ \text { H9 } & -0.7348000000 & -2.1237000000 & -0.2715000000 \\ \text { H10 } & -2.2220000000 & 1.5181000000 & -0.7655000000 \\ \text { H11 } & -2.3566000000 & -0.2050000000 & -0.2733000000 \\ \text { H12 } & -1.5581000000 & 0.1901000000 & -1.7792000000 \\ \text { C13 } & 2.0813000000 & -3.0517000000 & 0.6483000000 \\ \text { O14 } & 3.7293000000 & -1.4385000000 & 1.3172000000 \\ \text { H15 } & 2.8615000000 & -3.7450000000 & 0.9762000000 \\ \text { H16 } & 1.8632000000 & -3.1900000000 & -0.4143000000 \\ \text { H17 } & 1.1392000000 & -3.2585000000 & 1.1723000000 \\ \text { H18 } & 1.3790000000 & 1.3657000000 & 1.1725000000 \\ \text { I19 } & -2.6061000000 & -3.1588000000 & 0.9142000000\end{array}$

\section{(f)}

$\begin{array}{lrrr}\text { C1 } & -0.7354000000 & 0.1968000000 & 0.0092000000 \\ \text { C2 } & 0.7274000000 & -0.1653000000 & -0.0344000000 \\ \text { H3 } & -0.8949000000 & 1.0911000000 & -0.5946000000 \\ \text { H4 } & -1.0607000000 & 0.3741000000 & 1.0401000000\end{array}$




$\begin{array}{lrrr}\text { H5 } & -1.3502000000 & -0.6276000000 & -0.3683000000 \\ \text { O6 } & 1.5487000000 & 0.5078000000 & -0.6859000000 \\ \text { C7 } & 1.1566000000 & -1.3755000000 & 0.7423000000 \\ \text { C8 } & 2.8040000000 & -3.0315000000 & 1.5803000000 \\ \text { O9 } & 0.0820000000 & -1.9400000000 & 1.3773000000 \\ \text { C10 } & 2.4486000000 & -1.8413000000 & 0.7993000000 \\ \text { O11 } & 3.9268000000 & -3.5313000000 & 1.7012000000 \\ \text { O12 } & 1.7157000000 & -3.6390000000 & 2.2492000000 \\ \text { H13 } & 2.0237000000 & -4.4234000000 & 2.7488000000 \\ \text { H14 } & 0.3573000000 & -2.7413000000 & 1.8938000000 \\ \text { C15 } & 3.6116000000 & -1.1908000000 & 0.0816000000 \\ \text { H16 } & 4.5194000000 & -1.7631000000 & 0.2787000000 \\ \text { H17 } & 3.7601000000 & -0.1578000000 & 0.4102000000 \\ \text { H18 } & 3.4426000000 & -1.1424000000 & -0.9980000000\end{array}$

\section{(f) cluster}

$\begin{array}{lrrr}\text { C1 } & -0.7354000000 & 0.1968000000 & 0.0092000000 \\ \text { C2 } & 0.7314000000 & -0.1663000000 & -0.0345000000 \\ \text { H3 } & -0.8949000000 & 1.0992000000 & -0.5841000000 \\ \text { H4 } & -1.0607000000 & 0.3572000000 & 1.0429000000 \\ \text { H5 } & -1.3462000000 & -0.6265000000 & -0.3769000000 \\ \text { O6 } & 1.5461000000 & 0.5280000000 & -0.6813000000 \\ \text { C7 } & 1.1432000000 & -1.3767000000 & 0.7232000000 \\ \text { C8 } & 2.6518000000 & -3.0786000000 & 1.6048000000 \\ \text { O9 } & 0.0744000000 & -1.9724000000 & 1.3475000000 \\ \text { C10 } & 2.4292000000 & -1.8533000000 & 0.8003000000 \\ \text { O11 } & 3.9111000000 & -3.4974000000 & 1.6446000000 \\ \text { O12 } & 1.6976000000 & -3.6688000000 & 2.2028000000 \\ \text { H13 } & 4.1255000000 & -4.3537000000 & 2.1954000000 \\ \text { H14 } & 0.4307000000 & -2.7883000000 & 1.8427000000 \\ \text { C15 } & 3.6135000000 & -1.2117000000 & 0.1228000000 \\ \text { H16 } & 4.5106000000 & -1.7852000000 & 0.3538000000 \\ \text { H17 } & 3.7504000000 & -0.1745000000 & 0.4477000000 \\ \text { H18 } & 3.4807000000 & -1.1673000000 & -0.9638000000 \\ \text { I19 } & 5.1555000000 & -6.1645000000 & 3.2471000000\end{array}$

$\begin{array}{cccc}\text { (g) } & & & \\ \text { C1 } & -0.4148000000 & 0.6649000000 & -0.0252000000 \\ \text { C2 } & 0.4444000000 & -0.5194000000 & 0.0477000000 \\ \text { O3 } & -0.0417000000 & 1.7853000000 & 0.4491000000 \\ \text { C4 } & -1.7725000000 & 0.5609000000 & -0.6747000000 \\ \text { H5 } & 3.0516000000 & -3.4076000000 & 0.4564000000 \\ \text { O6 } & 2.1636000000 & 0.7642000000 & 1.1816000000 \\ \text { C7 } & 1.7003000000 & -0.4196000000 & 0.6488000000 \\ \text { N8 } & -0.0075000000 & -1.7107000000 & -0.4797000000 \\ \text { C9 } & 2.6468000000 & -1.5241000000 & 0.7810000000 \\ \text { H10 } & 0.6296000000 & -2.5060000000 & -0.4036000000 \\ \text { H11 } & -0.9068000000 & -1.7816000000 & -0.9189000000\end{array}$




$\begin{array}{lrrr}\text { H12 } & -2.2559000000 & 1.5377000000 & -0.6351000000 \\ \text { H13 } & -2.4042000000 & -0.1716000000 & -0.1554000000 \\ \text { H14 } & -1.6878000000 & 0.2463000000 & -1.7230000000 \\ \text { C15 } & 3.9606000000 & -1.2149000000 & 1.4605000000 \\ \text { N16 } & 2.3214000000 & -2.7010000000 & 0.3159000000 \\ \text { H17 } & 4.5874000000 & -2.1108000000 & 1.5057000000 \\ \text { H18 } & 3.7932000000 & -0.8393000000 & 2.4752000000 \\ \text { H19 } & 4.4975000000 & -0.4252000000 & 0.9246000000 \\ \text { H20 } & 1.4634000000 & 1.4716000000 & 1.0528000000\end{array}$

\section{(g) cluster}

$\begin{array}{lccc}\text { C1 } & -0.4148000000 & 0.6649000000 & -0.0252000000 \\ \text { C2 } & 0.4630000000 & -0.5451000000 & 0.0493000000 \\ \text { O3 } & 0.1521000000 & 1.7810000000 & -0.1627000000 \\ \text { C4 } & -1.9160000000 & 0.5824000000 & 0.0142000000 \\ \text { H5 } & 2.6538000000 & -3.7805000000 & 0.0344000000 \\ \text { O6 } & -1.3336000000 & -2.0622000000 & 0.5834000000 \\ \text { C7 } & 0.0050000000 & -1.8364000000 & 0.2558000000 \\ \text { N8 } & 1.7890000000 & -0.2617000000 & -0.1224000000 \\ \text { C9 } & 0.8729000000 & -3.0072000000 & 0.2295000000 \\ \text { H10 } & 2.4321000000 & -1.0551000000 & -0.1303000000 \\ \text { H11 } & 2.0301000000 & 0.7022000000 & -0.2942000000 \\ \text { H12 } & -2.3146000000 & 1.5942000000 & -0.0943000000 \\ \text { H13 } & -2.2617000000 & 0.1231000000 & 0.9442000000 \\ \text { H14 } & -2.2984000000 & -0.0627000000 & -0.7864000000 \\ \text { C15 } & 0.1888000000 & -4.3466000000 & 0.3972000000 \\ \text { N16 } & 2.1692000000 & -2.8752000000 & 0.0531000000 \\ \text { H17 } & 0.9258000000 & -5.1575000000 & 0.3889000000 \\ \text { H18 } & -0.5397000000 & -4.5106000000 & -0.4085000000 \\ \text { H19 } & -0.3762000000 & -4.3750000000 & 1.3349000000 \\ \text { H20 } & -1.8756000000 & -2.4545000000 & -0.1767000000 \\ \text { I21 } & -3.1232000000 & -3.2939000000 & -2.1400000000\end{array}$

(h)

$\begin{array}{lccc}\text { C1 } & -0.4529000000 & 0.5947000000 & -0.0104000000 \\ \text { C2 } & 0.4627000000 & -0.6327000000 & 0.0002000000 \\ \text { H3 } & -0.2235000000 & 1.3153000000 & -0.8118000000 \\ \text { O4 } & -1.3926000000 & 0.7626000000 & 0.7654000000 \\ \text { O5 } & 2.9887000000 & -2.0723000000 & -0.3858000000 \\ \text { C6 } & -0.2312000000 & -1.8218000000 & 0.6687000000 \\ \text { C7 } & 1.8206000000 & -0.2583000000 & 0.7232000000 \\ \text { O8 } & 0.7021000000 & -0.8611000000 & -1.4165000000 \\ \text { C9 } & 2.7070000000 & -1.4927000000 & 0.8326000000 \\ \text { O10 } & 1.4975000000 & 0.1742000000 & 2.0618000000 \\ \text { C11 } & 2.5860000000 & 0.8704000000 & 0.0060000000 \\ \text { O12 } & 3.1488000000 & -1.9436000000 & 1.8918000000 \\ \text { H13 } & 3.5804000000 & -2.8499000000 & -0.2798000000 \\ \text { H14 } & 3.5657000000 & 1.0117000000 & 0.4729000000\end{array}$




$\begin{array}{lrrr}\text { H15 } & 2.0256000000 & 1.8011000000 & 0.1213000000 \\ \text { H16 } & 2.7112000000 & 0.6503000000 & -1.0552000000 \\ \text { H17 } & 1.9601000000 & -0.3885000000 & 2.7210000000 \\ \text { H18 } & 1.3843000000 & -1.5576000000 & -1.5262000000 \\ \text { H19 } & 0.3934000000 & -2.7216000000 & 0.6296000000 \\ \text { H20 } & -1.1682000000 & -2.0347000000 & 0.1488000000 \\ \text { H21 } & -0.4602000000 & -1.5853000000 & 1.7098000000\end{array}$

(h) cluster

$\begin{array}{lrrr}\text { C1 } & -0.4529000000 & 0.5947000000 & -0.0104000000 \\ \text { C2 } & 0.4572000000 & -0.6254000000 & 0.0001000000 \\ \text { O3 } & -1.3852000000 & 0.7912000000 & 0.7731000000 \\ \text { O4 } & 2.9181000000 & -2.1147000000 & -0.5088000000 \\ \text { C5 } & -0.1990000000 & -1.8017000000 & 0.7263000000 \\ \text { C6 } & 1.8490000000 & -0.2483000000 & 0.6400000000 \\ \text { O7 } & 0.6449000000 & -0.9073000000 & -1.4179000000 \\ \text { C8 } & 2.7762000000 & -1.4727000000 & 0.5532000000 \\ \text { O9 } & 1.6151000000 & 0.1159000000 & 2.0180000000 \\ \text { C10 } & 2.5379000000 & 0.9290000000 & -0.0781000000 \\ \text { O11 } & 3.3913000000 & -1.7076000000 & 1.7106000000 \\ \text { H12 } & -0.2331000000 & 1.3032000000 & -0.8276000000 \\ \text { H13 } & 4.0825000000 & -2.5018000000 & 1.7903000000 \\ \text { H14 } & 0.4316000000 & -2.6938000000 & 0.6590000000 \\ \text { H15 } & -1.1635000000 & -2.0248000000 & 0.2620000000 \\ \text { H16 } & -0.3611000000 & -1.5504000000 & 1.7763000000 \\ \text { H17 } & 1.4162000000 & -1.5244000000 & -1.4910000000 \\ \text { H18 } & 2.2550000000 & -0.3890000000 & 2.5703000000 \\ \text { H19 } & 3.5509000000 & 1.0546000000 & 0.3179000000 \\ \text { H20 } & 1.9814000000 & 1.8491000000 & 0.1205000000 \\ \text { H21 } & 2.5890000000 & 0.7552000000 & -1.1552000000 \\ \text { I22 } & 5.6107000000 & -4.0521000000 & 2.4217000000\end{array}$

(i)

$\begin{array}{lccc}\text { O1 } & -0.5132000000 & -0.3931000000 & 0.0000000000 \\ \text { C2 } & 0.6410000000 & 0.4910000000 & 0.0000000000 \\ \text { O3 } & 1.4735000000 & 0.2531000000 & 1.1458000000 \\ \text { C4 } & 2.0923000000 & -1.0804000000 & 1.1770000000 \\ \text { O5 } & 1.1607000000 & -2.0875000000 & 1.5461000000 \\ \text { H6 } & -1.0953000000 & -0.1907000000 & 0.7635000000 \\ \text { C7 } & 0.2229000000 & 1.9649000000 & 0.0226000000 \\ \text { H8 } & 1.1563000000 & 0.2647000000 & -0.9364000000 \\ \text { C9 } & 2.7077000000 & -0.9791000000 & 3.6795000000 \\ \text { C10 } & 3.1751000000 & -1.0111000000 & 2.2539000000 \\ \text { H11 } & 2.5486000000 & -1.2703000000 & 0.1978000000 \\ \text { H12 } & 0.6413000000 & 2.5223000000 & 2.0447000000 \\ \text { H13 } & -1.0179000000 & 1.9897000000 & 1.7959000000 \\ \text { H14 } & -0.5186000000 & 3.5898000000 & 1.1895000000 \\ \text { H15 } & 3.5651000000 & -0.8998000000 & 4.3492000000\end{array}$




$\begin{array}{lrrr}\text { H16 } & 2.1349000000 & -1.8859000000 & 3.9008000000 \\ \text { O17 } & 0.2284000000 & 2.6016000000 & -1.0385000000 \\ \text { H18 } & 2.0321000000 & -0.1293000000 & 3.8309000000 \\ \text { C19 } & -0.1967000000 & 2.5584000000 & 1.3410000000 \\ \text { O20 } & 4.3647000000 & -0.9745000000 & 1.9132000000 \\ \text { H21 } & 0.4458000000 & -2.1316000000 & 0.8731000000\end{array}$

$\begin{array}{lccc}\text { (i) cluster } & & & \\ \text { O1 } & -0.5132000000 & -0.3931000000 & 0.0000000000 \\ \text { C2 } & 0.6127000000 & 0.4693000000 & 0.0000000000 \\ \text { O3 } & 1.4612000000 & 0.3370000000 & 1.1703000000 \\ \text { C4 } & 2.2209000000 & -0.8877000000 & 1.3558000000 \\ \text { O5 } & 1.4449000000 & -2.0040000000 & 1.7519000000 \\ \text { H6 } & -0.9364000000 & -0.4558000000 & 0.9037000000 \\ \text { I7 } & -1.6080000000 & -0.8965000000 & 3.4049000000 \\ \text { C8 } & 0.1778000000 & 1.9390000000 & -0.0131000000 \\ \text { H9 } & 1.1719000000 & 0.2499000000 & -0.9150000000 \\ \text { C10 } & 2.7266000000 & -0.3386000000 & 3.8291000000 \\ \text { C11 } & 3.2477000000 & -0.5041000000 & 2.4267000000 \\ \text { H12 } & 2.7437000000 & -1.1628000000 & 0.4337000000 \\ \text { H13 } & 0.4900000000 & 2.6496000000 & 1.9707000000 \\ \text { H14 } & -1.0508000000 & 1.8465000000 & 1.7637000000 \\ \text { H15 } & -0.8085000000 & 3.4921000000 & 1.0718000000 \\ \text { H16 } & 3.4760000000 & 0.1599000000 & 4.4473000000 \\ \text { H17 } & 2.5038000000 & -1.3239000000 & 4.2559000000 \\ \text { O18 } & 0.2422000000 & 2.5820000000 & -1.0755000000 \\ \text { H19 } & 1.7833000000 & 0.2178000000 & 3.8307000000 \\ \text { C20 } & -0.3436000000 & 2.5240000000 & 1.2703000000 \\ \text { O21 } & 4.4444000000 & -0.3629000000 & 2.1220000000 \\ \text { H22 } & 0.6590000000 & -1.7384000000 & 2.3084000000\end{array}$

(j)

$\begin{array}{lccc}\text { C1 } & 0.6935000000 & 0.2567000000 & 0.0580000000 \\ \text { C2 } & -0.7081000000 & -0.2775000000 & 0.0000000000 \\ \text { O3 } & 0.9673000000 & 1.4517000000 & -0.1430000000 \\ \text { O4 } & 2.6021000000 & 3.5983000000 & 1.3287000000 \\ \text { C5 } & 1.8055000000 & -0.7535000000 & 0.3556000000 \\ \text { H6 } & -0.7797000000 & -1.0108000000 & -0.8106000000 \\ \text { H7 } & -1.4101000000 & 0.5412000000 & -0.1635000000 \\ \text { H8 } & -0.9601000000 & -0.8075000000 & 0.9267000000 \\ \text { C9 } & 3.1989000000 & -0.1482000000 & 0.1180000000 \\ \text { O10 } & 1.5712000000 & -1.8731000000 & -0.5311000000 \\ \text { H11 } & 1.7323000000 & -1.0597000000 & 1.4113000000 \\ \text { C12 } & 4.3611000000 & -1.1496000000 & 0.2275000000 \\ \text { O13 } & 3.4480000000 & 0.8625000000 & 1.1599000000 \\ \text { H14 } & 3.2347000000 & 0.3249000000 & -0.8743000000 \\ \text { C15 } & 5.7462000000 & -0.5888000000 & 0.3560000000 \\ \text { S16 } & 3.5889000000 & 2.5792000000 & 0.5650000000\end{array}$




$\begin{array}{llrr}\mathrm{O} 17 & 4.1336000000 & -2.3740000000 & 0.1923000000 \\ \mathrm{H} 18 & 6.4759000000 & -1.3967000000 & 0.2883000000 \\ \mathrm{H} 19 & 5.8555000000 & -0.0641000000 & 1.3114000000 \\ \mathrm{H} 20 & 5.9353000000 & 0.1556000000 & -0.4274000000 \\ \mathrm{H} 21 & 2.2715000000 & -2.5441000000 & -0.3567000000 \\ \mathrm{O} 22 & 5.1983000000 & 2.8045000000 & 1.3437000000 \\ \mathrm{O} 23 & 3.8231000000 & 2.5637000000 & -1.0279000000 \\ \mathrm{H} 24 & 5.1140000000 & 3.5995000000 & 1.9288000000\end{array}$

\begin{tabular}{cccc}
\multicolumn{2}{c}{ lonized (j) } & & \\
C1 & 0.6935000000 & 0.2567000000 & 0.0580000000 \\
C2 & -0.7231000000 & -0.2832000000 & -0.0006000000 \\
O3 & 1.6677000000 & -0.4931000000 & 0.2055000000 \\
O4 & -1.3096000000 & 5.3227000000 & -2.6723000000 \\
C5 & 0.8234000000 & 1.7725000000 & 0.0026000000 \\
H6 & -1.0037000000 & -0.3941000000 & -1.0538000000 \\
H7 & -1.4444000000 & 0.3898000000 & 0.4757000000 \\
H8 & -0.7556000000 & -1.2656000000 & 0.4773000000 \\
C9 & 0.1570000000 & 2.4570000000 & -1.2194000000 \\
O10 & 2.2079000000 & 2.1650000000 & 0.0681000000 \\
H11 & 0.2754000000 & 2.1633000000 & 0.8781000000 \\
C12 & 0.6306000000 & 1.9188000000 & -2.5697000000 \\
O13 & 0.4701000000 & 3.8617000000 & -1.0888000000 \\
H14 & -0.9333000000 & 2.3160000000 & -1.1549000000 \\
C15 & 1.1519000000 & 2.9043000000 & -3.5762000000 \\
S16 & -1.0970000000 & 4.9860000000 & -1.0859000000 \\
O17 & 0.5379000000 & 0.6982000000 & -2.8055000000 \\
H18 & 0.4312000000 & 3.7196000000 & -3.7137000000 \\
H19 & 1.3593000000 & 2.3928000000 & -4.5185000000 \\
H20 & 2.0660000000 & 3.3710000000 & -3.1896000000 \\
H21 & 2.2082000000 & 3.1386000000 & -0.0770000000 \\
O22 & -0.5920000000 & 6.2102000000 & -0.1439000000 \\
O23 & -2.2195000000 & 3.9754000000 & -0.4419000000
\end{tabular}

